\title{
UN ENSAYO SOBRE LA EVOLUCIÓN DE LA GEOGRAFÍA URBANA EN ESPAÑA: REINVENTAR EL ESTUDIO DE LA CIUDAD
}

\author{
Andrés Precedo Ledo \\ Universidad de Santiago de Compostela \\ andresjose.precedo@use.es
}

\begin{abstract}
Resumen: Este trabajo constituye un ensayo crítico sobre la evolución de los estudios de geografía urbana en España. Parte de la prolongada experiencia docente, investigadora y profesional del autor como base justificativa de un discurso original que pretende aportar un nuevo punto de equilibrio a los estudios urbanos entre el desarrollo teórico y metodológico y los avances de las técnicas instrumentales. La discusión aporta una síntesis del proceso de innovación de la geografía urbana entre 1970 a 1990 y una proposición acerca de cómo los conocimientos teóricos acumulados pueden aportar una visión renovada al estudio y diseño de la ciudad del siglo XXI.
\end{abstract}

Palabras clave: geografía urbana, método geográfico, filosofía urbana, posicionamiento urbano, reinvención estudios urbanos, globalización.

\begin{abstract}
This paper is a critical essay on the evolution of urban geography studies in Spain. The long teaching, researching and professional author's experience is the basis of a speech, supporting the original aims to provide a new point of equilibrium for urban studies between the theoretical and methodological advances and the instrumental techniques. This discussion provides a summary of the innovations process of urban geography from 1970 to 1990 and suggests a new conceptual framework to the urban geographical studies inside a valorisation process to design in the new international urban system from a humanistic and social perspective.
\end{abstract}

Keywords: urban geography, geographic method, urban philosophy, urban positioning, reinvention urban studies, globalization. 
No pretendo en esta colaboración hacer un análisis académico acerca de la evolución de la Geografía Urbana como el propio título sugiere. Lo único que persigo es hacer una reflexión en voz alta acerca de mi percepción del estado actual de la disciplina a la que he dedicado tanto tiempo y trabajo desde la perspectiva de la geografía y de los estudios urbanos en España. Es por esa razón por la que -frente al estilo académico convencional del reflexivo impersonal- he optado por un estilo personal -no personalista- para significar dos cosas: la intención de establecer desde el principio un diálogo abierto con el lector, pero también porque toda la argumentación que sigue procede de la propia experiencia docente, investigadora y profesional. Nada por tanto más alejado de cualquier afán protagonista que, a estas alturas, resultaría al menos fútil e innecesario. De todas maneras a quien de otro modo lo interprete le ruego lo disculpe.

Puesto en esa tesitura, la primera valoración que me viene a la mente es la consideración de los estudios de geografía urbana en estos últimos años como una parte de la geografía que ha perdido peso y potencial de innovación en el contexto académico e investigador. Las frecuentes lecturas parecen confirmar esta idea, porque tanto los temas tratados como el modo de abordarlos no son más que una prolongación en el tiempo de las cuestiones, las técnicas y los métodos que en la etapa anterior se habían avanzado; tal vez con un aparato tecnológico más rico pero con un predominio de la técnica sobre la filosofía. Es más, no deja de resultar paradójico que mientras avanzamos hacia un mundo de ciudades, hacia una sociedad más urbanizada, hacia un nuevo orden económico mundial en el que el sistema de ciudades constituye su soporte físico preferencial, el discurso geográfico se mantenga en un estado de aletargamiento en general, sin profundizar ni defender las importantes aportaciones que desde la Geografía Urbana se pueden hacer para la construcción de una sociedad mejor y de unas ciudades más humanas. Y esta percepción me sorprende aún más cuando los propios geógrafos vamos asumiendo las aportaciones de otras disciplinas o técnicas sin someterlas a la crítica conceptual rigurosa que toda asunción de contenidos y principios exógenos requiere. Por todo ello, y por otras valoraciones que se podrían añadir, la Geografía Urbana que en los años setenta había sido la que con más intensidad contribuyó a la evolución y modernización de la geografía y a su conformación como disciplina teórica y científica de fuerte impacto social y económico, se haya ido replegando a un papel secundario en el cual el propio formato de los congresos nacionales, concebidos como un superficial itinerario urbano, sea tal vez su más evidente manifestación. Por eso, retomando una expresión de uso cada vez más frecuente, como es la reinvención de la ciudad, tal vez los geógrafos interesados por los fenómenos urbanos y preocupados por contribuir a través de ellos a la transformación de la sociedad, deberíamos empezar a pensar en la necesidad de reinventar también la geografía urbana. En lugar de limitarnos al seguimiento de las novedades con una ausencia demasiado generalizada de espíritu crítico deberíamos hacer una reflexión sobre le papel de la disciplina en el nuevo contexto 
urbano mundial. Dicho esto, ya podría terminar mi intervención porque el tema central de mi participación aquí no es otra que el suscitar entre los jóvenes la misma inquietud que a nosotros, cuando empezamos, nuestros maestros supieron transmitirnos.

No puedo dejar de recordar mis primeras incursiones en la cuestión urbana más allá de las aulas. Unas veces fue la participación en estudios socioeconómicos regionales o en planes de desarrollo regional como antes se denominaban, y en los cuales la red urbana y la organización funcional del espacio emergía como una conceptualización innovadora del territorio, al que aportaba nuevas categorías de intervención; otras veces era la participación en debates abiertos a la sociedad sobre el futuro de las ciudades o de una ciudad concreta; también la participación en cursos o seminarios destinados a la formación de futuros gestores políticos de variado espectro ideológico que en la visión geográfica de la ciudad encontraban un punto de encuentro o de desencuentro en algunas ocasiones, pero siempre de debate; recuerdo también mi participación en planes de urbanismo de ámbito general o parcial e incluso en concursos de diseño urbano o en la revitalización de los centros históricos; no puedo tampoco olvidar mis siete años de clase a los estudiantes de arquitectura para ayudarles a profundizar en el significado histórico, cultural y social del espacio urbano sobre el cual ellos habrían de intervenir; asimismo, la participación en planes de ordenación territorial en los cuales las relaciones urbanorurales constituían una parte importante e innovadora, en la constitución de las áreas metropolitanas, o en los estudios para la localización de las actividades económicas en la ciudad destinadas a las redes logísticas o de distribución de bienes y de servicios de grandes empresas o de pequeñas iniciativas comerciales. No quiero dejar de citar las oportunidades que la profesión me brindó para diseñar el modelo de ciudad para casos concretos de ciudades intermedias o para participar en institutos económicos para completar la visión sectorial con el componente espacial y urbano. Citaré también la preparación de planes de marketing urbano y de planificación participativa así como en el papel de las redes urbanas intermedias en el reequilibrio territorial de las regiones europeas y su vinculación con los modelos de desarrollo local. Probablemente podría añadir a estos ejemplos otros más, pero no voy a rebuscar en mi curriculum más aportaciones, principalmente porque no serían necesarias ya que en este comentario no pretendo otra cosa que exponer, a través de mi propia experiencia, las muchas posibilidades que la formación profunda en geografía urbana puede aportar a quienes se acerquen como yo mismo hice en su día, a esta disciplina geográfica de fuerte contenido aplicado. Y tampoco lo voy a hacer porque, a pesar de tan dilatada experiencia, lo que más grabado ha quedado en mi mente ha sido lo que aprendí en las lecturas sobre la evolución histórica de la ciudad, en el estudios de los reformadores sociales del siglo XIX, cuyos libros originales busqué acaloradamente para poder leerlos en su versión primera sin interpretaciones adulteradas o reduccionistas, o en las teorías que algunos sociólogos 
o algunos arquitectos habían elaborado sobre la ciudad que en cada momento era y la que debía ser en el mañana por venir. Siempre me sorprendió comprobar a través de sus escritos que los problemas eran casi siempre los mismos y que las preocupaciones también, porque en cada caso y desde filosofías diferentes o incluso antagónicas, la principal preocupación era siempre crear un estado del pensamiento urbano favorable a la persona en su dimensión individual y social, en su desarrollo evolutivo y en su ser cognitivo o existencial, en su acción económica o política, o en un pensamiento marcado por la imaginación creativa. En una palabra en el pensamiento urbano. Por eso, me duele cuando bajo el epígrafe de la teoría, lo que se enseña es un compendio de técnicas, de métodos, de sistemas de clasificación o de otro tipo de contenidos, carentes del pensamiento filosófico o de valores que es lo que verdaderamente da soporte a una buena teoría científica de carácter social como la nuestra. Probablemente esos congresos itinerantes a los que antes me referí, aún cuando aporten una revisión fugaz de los cambios recientes de las ciudades españolas- sean el resultado de la actual banalización de los estudios geográficos sobre la ciudad y la sociedad urbana.

Pero no termina aquí mi reflexión general sobre el papel de la Geografía Urbana, porque a pesar del interés del pensamiento, la preparación para la acción práctica es un instrumento fundamental para trasladar las ideas a la realidad, a esa realidad que está en nuestras manos transformar o dirigir, aunque sea en tareas de aparente poca importancia. Porque toda acción que contribuya a mejorar o prepara el futuro, a la escala que sea, es siempre tarea de notable trascendencia y portadora de no pocas satisfacciones personales y sociales: pero, para que así sea es preciso una acción reflexiva y critica antecedente, porque la aplicación de técnicas sin ideas que las soporten no dejan de ser otra cosa que una instrumentación operativa vacía de contenido, y que probablemente nunca pasará del papel en que se escribió. Es cierto que el formato actual de los planes de estudio y el concepto tecnocrático de una universidad burocratizada y pragmática, no facilitan el desarrollo del pensamiento crítico, pero más allá de tan escueto contenedor, el individuo mismo dispone de todos lo medios necesarios para hacer trabajar sus capacidades intelectuales fuera de tan limitantes ámbitos. Y esa es tarea del profesor de geografía urbana que quiera pasar de ser un enseñante para convertirse en un maestro. Tal vez ese pueda ser el camino para reinventar la geografía urbana y repensarla desde una nueva perspectiva, en función de los diferentes ciclos económicos o como sería mejor decir, de civilización.

\section{La recuperación de la perspectiva}

Las ciencias sociales en el siglo XX han experimentado una evolución común. Inicialmente constituían ese necesario escalón entre las ciencias experimentales y las 
ciencias filosóficas, es decir eran disciplinas que combinaban e integraban en síntesis explicativas la teoría y la práctica, con una orientación aplicada para contribuir a la resolución de muchos de los problemas sociales de la comunidad. Por eso eran disciplinas basadas en la observación atenta, en la explicación rigurosa, y en una praxis posibilista y siempre con un pensamiento teórico, ideológico o político detrás. Naturalmente de cual fuera este substrato dependerían los enfoques y los resultados que en no pocos casos terminarían en manifiestos cargados de ideología pero vacíos de ciencia. En cualquier caso, el tiempo y la crítica interna y externa terminarían por decantar las aportaciones, extrayendo de cada una de ellas lo más valioso para la conformación del corpus teórico. También la Geografía Urbana, como la geografía humana en general, experimentó ese proceso, solo que con mayor frecuencia e intensidad que en otras ciencias sociales el debate se orientó hacia una crítica destructiva de los aportes anteriores, de manera que cada paradigma sustituía al anterior, superponiéndose a él, en lugar de complementarlo como hubiera sido deseable, y también razonable. Fue así como unas veces de la mano del neopositivismo el empirismo, tantas veces limitado a su aspecto formal y técnico, renunció a los contenidos de la llamada geografía urbana clásica acusándola de descriptiva, aplicando este término como sinónimo de ineficaz, ya que el utilitarismo se convertiría en la razón de ser y la descripción no aportaba nada a la acción que era lo que se pretendía. Unas veces esa preeminencia de la acción se pensaba desee el pragmatismo utilitarista de la planificación, olvidando que la filosofía del plan constituye la parte más valiosa del mismo; y otras veces a la acción social, como actitud proactiva para construir una nueva sociedad desde la visión de una contestada y pronto superada doctrina política. De este modo, la Geografía Urbana heredada de la primera mitad del siglo pasado fue suplantada por la corriente teorético-cuantitativa del neopositivismo utilitarista unas veces, y por la corriente marxista o neomarxista que veía en la ciudad un espacio para el conflicto de clases, para la apropiación del valor del trabajo y de la tierra por el capital, y en consecuencia como un espacio de poder. La primera aportaba la lógica espacial y la segunda la lógica social, y aunque en el origen estaban sesgadas por sus doctrinas subyacentes , es decir el neoliberalismo capitalista y el marxismo radical, en su cierre de ciclo, una vez liberadas de los pronunciamientos primeros y de los fundamentalismo económicos y sociales, dejaron a la Geografía Urbana nuevos contenidos que se añadieron a los anteriores, aunque casi siempre presentados como una disociación excluyente. El problema de estas tensiones dialécticas fue que, por el camino de la confrontación y la crítica superficial, la geografía urbana fue sumiéndose, como gran parte de la geografía humana, en un nuevo reduccionismo tecnológico, cuando el deslumbramiento producido por las nuevas tecnologías llevaron a mirar a los instrumentos de análisis y de planificación como objetivos en sí mismo, olvidándose una vez más que los instrumentos sin las ideas, sin el pensamiento, no son más que una técnica sin principios ni valores.

Lo más paradójico de esta situación es que a medida que la Geografía Urbana se adentraba con inesperadas conversiones personales en el tecnologismo, otras temáti- 
cas que habían sido introducidas en el conocimiento científico por la geografía urbana clásica eran redescubiertas por otros profesionales, principalmente economistas, arquitectos, sociólogos o nuevas profesiones, y -reinventándolas- se convertían en novedades que los geógrafos urbanos acogían con el entusiasmo de quien abierto solo a la novedad, desconoce su propio pasado. Y, mientras tanto, los planificadores urbanos que fueron pasando del urbanismo a la planificación estratégica y al marketing urbano, llegaron, por vía de agotamiento y a causa del mimetismo general, al descubrimiento de los valores, de los intangibles, como soportes de un nuevo modelo de ciudad inmaterial,. Ahora, la crisis del ciclo de las geografías nacionales y su superación por una geografía globalizada acentuó la necesidad de buscar nuevas fuentes de conocimiento para profundizar en los componentes esenciales del espacio urbano, a modo de una nueva metafísica social, ambiental y cultural en la que la geografía urbana puede encontrar una oportunidad. Para ello sería, no obstante, necesaria una revisión de todos sus contenidos para encontrar en ellos nuevos recursos que transformados en valores pudieran servir para pensar y proyectar un nuevo modelo de ciudad.

Lástima que, cuando examinamos lo que los geógrafos urbanos españoles están haciendo, nos encontramos que en una gran mayoría se ocupan de temas prestados en los cuales otros científicos y profesionales están en condiciones de aportar, en el mejor de los casos, lo mismo que nosotros pudiéramos hacer, pero cada uno un plus de especialización y experiencia del cual muchos geógrafos urbanos carecen. Basta con ver como los trabajos más frecuentes de los publicados tratan sobre ciudad y cambio climático, áreas metropolitanas, centros históricos, urbanización difusa o compacta, desarrollo local, sistema funcional de ciudades y jerarquías, estudio de ciudades medias y pequeñas y otros temas recurrentes. En todos ellos la Geografía Urbana ha entrado de la mano de otros especialistas, principalmente economistas y arquitectos, que fueron quienes actuaron como introductores de la innovación tanto en el mundo científico como en el ámbito de los programas oficiales. Cierto que no faltan los casos en que geógrafos urbanos hemos alcanzado posiciones preeminentes en alguno de esos campos compitiendo con ventaja con otros especialistas, pero esto constituye una excepcionalidad más vinculada a las oportunidades de las personas que a la propia trayectoria de la disciplina. A estos efectos contaré una anécdota acudiendo una vez más al diálogo personal:

Hace unos cuantos años asistí a un congreso sobre el urbanmarketing organizado por el economista creador de este concepto, recopilé el material disponible y a él añadí un informe que otro economista había realizado sobre el tema; como en esos momentos empezaba a redactar el texto del libro "Ciudad y Desarrollo Urbano" incorporé un capítulo sobre el tema, sin otra pretensión que aportar un visión actualizada del estudio de las ciudades desde la perspectiva de la planificación. Pasaron los años, y un día me encontré que un manual monográfico sobre esta materia me situaba 
como el introductor del marketing de ciudades en España, lo cual repitieron después otros especialistas. No es que tal afirmación no sea cierta, que lo es, pero mi papel no ha sido otro que el de transmisor de conocimiento, y el resultado una casual oportunidad. Otra cosa es que a partir de entonces no haya retomado el tema hasta elaborar un nuevo modelo, el urbanpositioning, que se diferencia del anterior en el punto de partida y en una concepción más geográfica, cultural y social que el modelo original, de perfil economicista como todos conocemos, y que, tal vez por eso, haya derivado en una mera estrategia de comunicación -el urbanbranding- la mayor parte de la veces aplicada a la promoción turística de las ciudades y los territorios.

He citado este ejemplo y lo he personalizado porque ilustra lo antedicho en primera persona, o sea el tomar temas de prestado, pero a diferencia de otros casos he intentado hacer una revisión teórica y metodológica desde la perspectiva geográfica, es decir desde una nueva perspectiva, lo cual no es más que una de tantas constataciones de aquello que nos enseñaban en la universidad, y de cuyo autor no recuerdo ahora el nombre: que la geografía avanza por sus márgenes. Ciertamente así es, pero no creo que tal tipo de avance sea privativo de la geografía, ya que la propia definición del término modelo, como construcción explicativa preparatoria de la teoría, esta basado en la incorporación de teorías particulares procedentes de otras disciplinas para incorporarlas a otro ámbitos del conocimiento que en cada caso sea objeto de su aplicación. Muchas de esas teorías que nos sirvieron de modelos se convirtieron entonces en punto de partida común para desarrollar nuevos conocimientos desde la perspectiva geográfica, y así llegar a construir y avanzar en nuestra propia teoría. Esto ha ocurrido con los modelos de gravedad que tan fructíferos han sido para la delimitación de áreas de influencia y de mercado, con la teoría general de sistemas aplicada al espacio interurbano y a la red urbana en particular o también al espacio intraurbano, o el darwinismo como inspirador de modelos y teorías sobre el crecimiento del espacio urbano, por no referirme más que a teorías generales. En todos los casos, el avance se ha producido por una nueva aportación al conocimiento geográfico a partir de la reelaboración de la teoría tomada como modelo y adaptada al pensamiento geográfico. Pero eso no es lo mismo que adoptar los postulados y los avances de otras disciplinas de manera mimética o repetitiva, motivados tan solo por el atractivo de la novedad, de la actualidad o de la moda. Estos factores pueden constituir estímulos para la innovación, y entonces la Geografía Urbana se enriquece y los geógrafos que a ella nos dedicamos podemos obtener mayor atención, al menos porque estamos en condiciones de aportar un valor o un punto de vista diferente: el geográfico; es decir por el valor de la diferenciación que tanto interés tiene en ámbitos diversos de un mundo de ciudades competitivo, reiterativo y monotemático. No debemos ser solo incorporadores de novedades, hemos de ser portadores de innovación.

El discurso anterior me trae a la cabeza el viejo tema, pero no por ello banal, de la perspectiva geográfica como factor diferenciador de la geografía. Ya sé que la cla- 
sificación cartesiana de las ciencias es un enfoque superado cuando es la multidisciplinariedad y la transversalidad lo que característica la ciencia actual, pero ello no impide que dentro de ese contexto cada científico, investigador o profesional debe tener nociones de la totalidad pero a la vez desarrollar su propia visión diferenciadora. Es ahí cuando debemos retomar la visión del geógrafo sobre la ciudad como elemento de diferenciación y como valor. No me canso de repetir a los alumnos que mientras los arquitectos, los economistas, los sociólogos, los administrativistas, los politólogos y otros especialistas tienen una visión fragmentaria de la ciudad, el geógrafo es capaz de aportar esa visión sintética que nos permite anticipar el impacto que cada nueva intervención puede tener en los restantes elementos de la ciudad, entendida como un sistema integrado o lo que viene a ser lo mismo interrelacionado e interactivo; y no solo en el plano funcional , también visualmente donde la relación del espacio urbano con el marco físico adquiere una relevancia notable. Recuerdo cuantas veces he redactado breves informes que han logrado paralizar o modificar importantes proyectos de actuación al hacer considerar al responsable político correspondiente del efecto negativo que dicha actuación, diseñada por arquitectos más o menos prestigiosos, iba a producir en el modelo de ciudad que el mismo se había planteado como objetivo. Otro caso semejante ocurre cuando se aborda el tratamiento de los centros históricos, porque una cosa es la rehabilitación constructiva, arquitectónica o del espacio público y otra muy diferente el metabolismo social y ambiental de la zona; y, en relación con esto, considerar que a menudo existen fuertes contradicciones entre los objetivos planteados para la recuperación de los centros históricos y las actuaciones pensadas para otras zonas de la ciudad. Esa capacidad que la visión de la Geografía Urbana nos aporta para comprender la ciudad como un todo en una foto mental instantánea pero no por eso menos reflexiva, porque procede de observaciones anteriores, constituye un valor diferenciador y potenciador de la importancia que la Geografía Urbana tiene para el estudio, la planificación y el diseño de la ciudad. Nada de eso lograremos si nos limitamos a repetir lo que otros han aportado desde su perspectiva, abandonando o incluso despreciando lo que nosotros podemos presentar como nuestro principal valor. Seguramente de tantas controversias, de tanto afán de novedad, de tanta falta de autoestima científica, hayamos devenido en esa adicción que algunos tienen a ser meros copiadores o transmisores de lo que otros han aportado, sin preocuparse de integrar esos conocimientos en el compendio teórico de la Geografía Urbana. Pero para hacer esto es necesario tener en la cabeza un constructo mental que actúe como filtro cognoscitivo para que lo que entra como conocimiento particular se transforme en conocimiento geográfico.

En resumen, del mismo modo que abandonando posiciones dogmáticas de ayer, y reorientando la senda hacia una visión comprehensiva y complementaria de todos los conocimientos que -desde la geografía o desde otras ciencias o praxis- han contribuido a enriquecer nuestro pensamiento científico y nuestra creatividad crítica, podemos alimentar la masa intelectual de nuestro pensamiento y perspectiva geográ- 
fica, esa visión geográfica básica es lo que valoriza nuestras percepciones y canaliza nuestra creatividad para llegar a obtener un compendio teórico diferenciado, y, por ello, especifico. De nuevo la vuelta a la perspectiva del geógrafo que nuestros maestros trataron de inculcarnos mediante su discurso académico. Recuerdo bien las intuitivas aportaciones de mi maestro Ferrer y las concienzudas y sapientes aseveraciones de Floristán, o la insistencia en la necesidad de buscar la explicación de la realidad a partir del principio de causalidad que mis clases de filosofía me aportaron, o la necesidad de buscar el punto de equilibrio que mis clases de teoría económica propinaron, pero por encima de todo ello no me olvidaré a aquél profesor de literatura que me enseñó a leer el Quijote haciendo una comparación entre los principios del arte barroco y los recursos lingüísticos del autor. Las sombras y las luces, los tiempos, los movimientos, las líneas curvas y rectas, y otros elementos del barroco podían identificarse en los versos o en la música de poetas y compositores. Ese descubrimiento fue para mí el arranque de la visión comparativa que más tarde volvería a encontrar en el hoy ya arrinconado método geográfico, y que tan felizmente habría de aplicar al estudio del plano o a la evolución histórica de la ciudad desde una perspectiva geográfica pero también filosófica y estética, porque estos valores no deben estar ausentes de quienes tenemos entre nuestros fines el estudio, la interpretación y la dinámica de los paisajes geográficos, aunque ahora parece que el tema de los paisajes urbanos lo hayamos postergado al baúl el pasado. He de confesar que, al menos a mí, eso fue lo que me atrajo cuando después de mis estudios de la economía, del derecho y de la empresa y de mis trabajos en contabilidad financiera opté por adentrarme en aquella disciplina que me permitiría interpretar lo que hasta entonces no había sido otra cosa que el objeto entusiasta de mi contemplación estética y visual, y a la vez aportar mi estudio a la mejora de las condiciones de vida de aquella sociedad que había creado, heredado o trasmitido esos paisajes que la geografía me presentaba. Otros muchos, de mi mano, encontraron también no poca satisfacción en ese modo de ver.

No quiero decir con esto que hayamos de volver al pasado ni recluirnos en romanticismos geográficos, solo pretendo hacer ver la que geografía en general y la Geografía Urbana en particular encierran en sí valores sustanciales para poder desarrollar un campo de conocimiento susceptible a de aportar al conjunto de las ciencias sociales, y a la aplicación social de los conocimientos, un renovado valor.

Pero para ello es necesario resolver antes la ecuación formada por el binomio teoría y praxis. Desde luego, como antes he dicho, la praxis sin teoría, la acción sin el pensamiento, no puede proporcionar un soporte firme a un conocimiento que quier ser original, creativo y a la vez capaz de ser aplicado a los problemas que surgen en las relaciones entre el hombre y el medio, y este caso entre el habitante urbano y el medio que lo rodera y que por eso constituye el marco de su ciclo vital, de su actividad cognoscitiva y de su inquietud proactiva y existencial. Es ahí donde debemos 
intervenir y por eso hemos de entrar desde un pensamiento bien acendrado y no solo desde la instrumentalidad de una técnica o de un método. Todo es importante pero el pensamiento-afirmo una vez más-debe preceder a la acción. Y el pensamiento se alimenta de lo que leemos en los libros o en la red, pero de una manera reflexiva y profunda. Tal vez de eso adolezca hoy la Geografía Urbana expuesta en las aulas en comparación con la que los mayores tuvimos la suerte de recibir. Pero toda oportunidad requiere esfuerzo para alcanzarla. Nosotros disponemos de un valor, de una idea-fuerza, de un factor diferenciador, de todo lo necesario para ser competitivos entre quienes se interesan por el fenómeno urbano.

\section{La reinvención de una geografía urbana disociada}

Tras las reflexiones anteriores acerca de la relación pensamiento-acción y de la validez de la visión geográfica de la ciudad como un valor añadido y diferenciador, me gustaría hacer un repaso breve de la evolución de la Geografía Urbana en el contexto de la geografía humana en particular y de la geografía en general. La idea que pretendo subrayar es que los estudios urbanos en geografía han actuado como motores de innovación para el conjunto de la geografía. No se trata en modo alguno de adoptar una perfectiva particularista y excluyente de las otras ramas, tan solo intento poner de manifiesto que frente al dinamismo de etapas anteriores, cuando los principales avances teóricos y metodológicos que a través de la Geografía Urbana se canalizaron hacia la teoría geográfica general, en la actualidad la Geografía Urbana se encuentra en una situación que podíamos calificar de aletargamiento. También es mi intención aportar una visión integradora de las diferentes aportaciones en un cuerpo común que denote el potencial que en el contexto actual tienen la perspectiva geográfica de la ciudad para elaborar una visión estratégica de posicionamiento o reposicionamiento en un contexto marcado por un cambio de ciclo a escala mundial Nada mejor para ello que repasar algunas aportaciones de la Geografía Urbana desde su propia evolución.

Podríamos situarnos en la segunda mitad del siglo pasado cuando en la geografía española se publican las primeras tesis doctorales sobre ciudades españolas (J. Bosque). Hasta entonces, nuestra geografía había sido principalmente una ciencia de lugares o una ciencia del paisaje en su formulación más elaborada heredera de la escuela francesa, pero con una fuerte presencia de los espacios rurales agrupados en conjuntos comarcales o regionales. Era una geografía donde el medio físico tenia un enorme peso, hasta el punto de convertirse en la práctica de un cierto neologismo geográfico que muchas veces fue más allá de lo que a la propia geografía corresponde, lo cual facilitó una primera disgregación de la geografía porque entre la Geografía Humana y Económica y la Geografía Física se había producido una enorme 
escisión analítica y metodológica. No fueron pocos los que entonces defendieron la necesidad de mantener la unidad del método geográfico pero con una visión tan neologista y naturalista que quienes veíamos en nuestra disciplina una ciencia social no teníamos otra suerte que apostar por esa disociación. Esto produjo, a mi modo de ver, un daño colateral por cuanto el estudio de los paisajes geográficos se circunscribió a los paisajes rurales. Por eso, si al principio la Geografía Urbana se esforzaba en buscar las relaciones existentes entre los factores del medio físico a través del estudio del emplazamiento y de la situación, pronto muchos geógrafos relegamos el papel del medio físico y nos centramos en la historia del plano, algunas veces con una orientación excesivamente historicista y con un método de investigación propio de los historiadores, que encontraban sus principales fuentes de documentación en los archivos y en los catastros. Naturalmente este enfoque mantuvo la escolar convergencia entre la geografía y la historia, no en vano muchos geógrafos de esa época tenían formación histórica, pero produjo una cierta disociación en la unidad del método geográfico. Al mismo tiempo, el estudio de lugares, es decir de ciudades o de barrios, fue alejando a la Geografía Urbana de la posibilidad de un propio cuerpo teórico. El marco existente iba poco más allá del estudio de la población y la descripción de las funciones sin criterio alguno de priorización y con total carencia de una visión estratégica. Por eso quienes seguimos otros caminos tuvimos que buscar la teoría en la historia del urbanismo que, como historia de la ciudad, aportaba al estudio el plano y de la morfología urbana un rango de conocimiento mucho más amplio y proporcionaba un modelo evolutivo de referencia para enmarcar el estudio de las ciudades particulares, siendo el análisis evolutivo del plano y de la morfología y los usos del suelo asociados a los cambios de la funcionalidad urbana un interesante sustituto. Paralelamente la Geografía Urbana española se adentró en la legislación urbanística de carácter administrativo para estudiar la evolución del plano urbano y para poner de relieve los factores políticos y económicos que estaban detrás de la desafortunada construcción de nuestros paisajes urbanos. Sea como fuere, el caso es que por la vía del historicismo o del urbanismo administrativista la Geografía Urbana fue descolgándose del estudio detenido del medio físico y de sus relaciones con la ciudad. Vistos desde una perspectiva actual esa disociación de lo fisiográfico y de lo paisajístico dejó en segundo término la importancia que los factores del emplazamiento o de la situación pueden aportar para el diseño de la ciudad, para la determinación de los usos del suelo, para el diseño del paisajismo urbano o para la identificación de recursos estratégicos útiles para generar ventajas comparativas en las que muchas ciudades pueden basar su propio esquema de competitividad y posicionamiento. Un nuevo valor que la geografía urbana puede aportar al estudio de las ciudades.

Un segundo paso importante se dio con la concepción geográfica de las relaciones campo-ciudad o mejor aún de la concepción conjunta de la ciudad y del territorio. Este enfoque constituyo sin duda una de las grandes aportaciones de la Geografía Urbana, enfatizando por primera vez en el mundo científico la relación entre las fun- 
ciones urbanas y el área funcional o de influencia de las ciudades, es decir de la influencia de la ciudad y el territorio circundante. Fueron los economistas espaciales, a los que más adelante me referiré. quienes a partir de esta invención, que siempre nos atribuyeron a los geógrafos, desarrollaron el análisis espacial y las teorías iniciales de la economía urbana.

Me gustaría destacar un tercer aspecto: el interés por los estudios comparados de ciudades a nivel europeo principalmente pero también a niveles más amplios. La idea, como el propio método geográfico clásico proponía, era llegar a la formulación de principios teóricos generales a partir de los estudios comparativos de los casos particulares. Cierto que, como la crítica posterior subrayó, el camino era muy lento y en la mayor parte de los casos, dichos estudios se quedaron en meras descripciones particularistas. Con todo, se hicieron notorios avances y se abrió un campo de gran interés, pero que también fue quedando postergado incluso si pensamos en ámbitos tan reducidos como los de nuestro propio país. Se decía que se trataba de una geografía descriptiva y por ello poco o nada científica. Ciertamente que en la mayoría de los casos era así, pero en otros la descripción del paisaje urbano o del espacio urbano en general esta asociada a una interpretación explicativa que, enmarcada en las teorías urbanísticas económicas y sociales existentes, permitía interesante avances para la comparativa urbana, pero también para conocer cuales eran los factores explicativos del desarrollo urbano en cada fase temporal y socioeconómica de los grandes ciclos evolutivos. Efectivamente, el descriptivismo es poco científico, al menos si no va más allá del planteamiento inicial, pero cuando la descripción es explicativa y sintética constituye un camino tan válido como cualquier otro para abordar el análisis de las ciudades desde la búsqueda de factores diferenciales en que sostener o implementar políticas o estrategias de desarrollo urbano. Máxime en una mundo urbano global como en el que nos encontramos. Aquí tenemos otro valor que la geografía urbana puede aportar a la sociedad actual.

Estos tres rasgos que, a modo de ejemplo, acabo de resumir sintetizan como la evolución de la Geografía Urbana en la primera etapa, la que denominamos con frecuencia clásica, fue abriendo caminos divergentes que aportaron a los estudios geográficos, centrados hasta entonces en la dilectancia geológica o en estudios formalistas de paisajes rurales, un hálito de innovación y modernidad. Lástima que fuese acompañado de una fuerza disociadora que solo en pocas ocasiones logró complementar la diversidad de los enfoques señalados en una síntesis integradora, tal como la propia naturaleza de la perspectiva geográfica exigiría. La experiencia primera es clara, cuando abandonamos la visión de síntesis y de relaciones, propia de nuestra disciplina y la que nos diferencia de las otras, por estudios temáticos que nos colocan en posición subsidiaria con respecto a las disciplinas de origen. Por este camino, tanto la geografía en general como la Geografía Urbana en particular pierden su ventaja comparativa, su razón de ser, y también su valor social. Independiente de ello, 
muchos geógrafos han logrado destacar en ámbitos relacionados con el nuestro pero colaterales, pero casi siempre con una presencia secundaria, o en todo caso con una vinculación particular a la persona.

La segunda etapa, como todos sabemos, la podemos situar en la España en los años setenta. Por un lado se produjo una importante ampliación del ámbito espacial de estudio de la Geografía Urbana y, por otro, una gran renovación metodológica e instrumental. Desde el primer punto de vista se introdujeron conceptos como la red urbana, la jerarquía urbana y el sistema de ciudades, una sucesión conceptual que encerraba detrás de sí una importante renovación epistemológica. El concepto de red urbana fue aplicado inicialmente como una ampliación de los temas propios de la metodología clásica de de los estudios regionales (Estébanez en su tesis sobre Cuenca) pero casi simultáneamente se constituyó como objeto especifico de estudio y soporte de unos estudios regionales renovados (Precedo con una tesis sobre la red urbana de Navarra) para ser más tarde aplicado a la totalidad de las ciudades españolas (Capel a la clasificación funcional de las ciudades y Diaz Nicolás para la jerarquía urbana); y con una visión más evolucionada del sistema urbano y de sus diversas fases evolutivas en el final de la etapa industrial y en la transición a la postindustrial (los estudios de Ferrer y Precedo para sucesivas comisiones de la U.G.I.). De este modo, se introdujo el análisis sistémico en la Geografía Urbana, lo cual aportó principalmente una perspectiva más integradora del espacio y una nueva estructura mental en la conceptualización del sistema territorial desde un enfoque dinámico. Por este camino la Geografía Urbana se articuló en tres grandes apartados: el sistema intraurbano para el estudio de la ciudad, el sistema interurbano para estudiar la red urbana y el sistema urbano como síntesis de los dos anteriores desde una perspectiva más amplia, enmarcada en el contexto socioeconómico, político y tecnológico en el que el procesos de urbanización se desarrollaba. Bajo esta perspectiva la Geografía Urbana adquirió un papel predominante en la ordenación del territorio por cuanto el sistema de ciudades pasó a ser el armazón en que los planes regionales se organizaron a partir de entonces, a menudo de la mano también de los economistas y de los arquitectos, produciéndose una convergencia temática y metodológica, aunque en cada caso priorizando objetivos diferentes y específicos de cada punto de vista.

Pero lo más importante fue la incorporación a la investigación y a la docencia geográfica del método científico experimental, porque la suma de la teoría general de sistemas y del método positivo del conocimiento científico cambió radicalmente el marco epistemológico anterior: lo que se llamó entonces la revolución teorético-cuantitativa. El cambio se produjo en todas las ciencias sociales pero en la geografía supuso una innovación mayor por cuanto el lenguaje estadístico-matemático y la modelización de los procesos le eran más ajenos. Fue entonces cuando la Geografía Urbana desempeñó con mayor nitidez su papel de foco innovador de la geografía 
humana, ese papel que en la actualidad ha dejado de ejercer. El paisaje geográfico convertido en espacio funcional supuso una gran evolución desde el punto de vista de la configuración de la Geografía Urbana como una disciplina aplicada a la planificación, abriendo nuevos caminos profesionales, pero también un reduccionismo del enfoque hacia un funcionalismo neopositivista, que si bien aportó un importante desarrollo teórico, más por importación de otras áreas que por creación propia, dio lugar a una pérdida de visión cultural y crítica, que siguió recayendo en los estudios urbanos tradicionales y más aún, con la llegada de la geografía radical como se llamó a la nueva versión del espacio desde la teoría marxista, y que tuvo su principal aportación en la teoría sobre la producción capitalista del espacio urbano (Capel con su conocido libro sobre la relación entre el capitalismo y la morfología urbana). Pero así como este enfoque no dejó de ser un nuevo marco interpretativo del crecimiento urbano, el análisis sistémico y la modelización, además de sus aportaciones teóricas que más arriba señalé, facilitó una internacionalización de los conocimientos y un avance de los estudios comparativos, basados ahora en el análisis de variables siguiendo definiciones operativas y técnicas comunes y compartidas, que facilitaron el estudio y comprensión de los procesos y su evolución en los distintos entornos Además así como en las primeras formulaciones funcionalistas la conceptualización prescindía de aquellos factores que no fueran medibles, las nuevas versiones incluyeron ya indicadores indirectos de los factores aleatorios y subjetivos de tipo cualitativo; y el propio modelo de las jerarquías urbanas verticales, que en algunos casos alcanzó una gran sofistificación matemática, se abrió a las jerarquías horizontales, más adecuadas para formalizar los sistemas urbanos surgidos de la primera internacionalización de los flujos económicos, conectivos y tecnológicos, derivados de la primera fase de la internacionalización geoeocnómica.

Estamos, por tanto, ante un desarrollo teórico de elevado valor para la planificación de las ciudades en la actual globalización, dado que la nueva formulación de las variables que conforman los sistemas urbanos significaron un importante punto de apoyo par los estudios y planes estratégicos en los cuales la internacionalización, la innovación y la competitividad de las ciudades constituyen componentes básicos, unidos a otras valores y trasversales como la sostenibilidad. Sin embargo, en general los geógrafos españoles, salvo excepciones (Precedo 1996 en su libro sobre Ciudad y Desarrollo Urbano) no prestaron demasiado interés a este tema que quedó en manos de los economistas. De todos modos, la Geografía Urbana esta todavía en condiciones de aportar una base teórica e interpretativa de los procesos de la que otras disciplinas carecen, ante la dificultad que estas tienen para adentrarse en el estudio de la complejidad de la ciudad en la que los factores del medio físico o ambientales, los urbanísticos, los sociales y los económico-funcionales constituyen necesariamente un todo integrado superador de la segmentación actual. Por eso la visión geográfica adquiere una nueva importancia tanto para definir la "visión de posicionamiento" que dichos estudios requieren, como para analizar la totalidad de los recursos urbanos, 
por cuanto todos constituyen posibles factores para identificar las ventajas comparativas de cada ciudad.

Evidentemente, si comparamos el discurso final de la etapa anterior con el que acabamos de exponer, nos damos cuenta que la Geografía Urbana entre 1960 y 1990 transitó por un nuevo camino que, en cierta manera, y principalmente en el análisis teórico cuantitativo, atrajo al resto de la áreas de estudio propias de la geografía humana, pero a esa tracción contribuyeron de manera muy eficaz las nuevas teorías espaciales que unas veces a través de los propios geógrafos, otras de los economistas pero también de los sociólogos, legaron un teoría de la ciudad y del espacio urbano mucho más amplía y consistente que la recibida de la etapa anterior. Este es el segundo aspecto del cambio a que antes me refería.

No dudo al afirmar que de todas las nuevas aportaciones la más importante fue la teoría del central place. El significado de la teoría de Christáller y la deficiente transmisión de la misma que ha tenido lugar en España, justifica un comentario específico sobre este tema. A menudo suele tratarse como un modelo anacrónico y excesivamente formalista basándose en el desarrollo expositivo de lo que el mismo denominó "principio de mercado". Pocos autores hacen referencia suficiente a los otros dos principios y menos aún se ha dado a conocer la formulación final del modelo, donde el autor superpone la organización espacial resultante de la aplicación de los tres principios simultáneamente, llegando así a una estructura jerárquica más flexible y compleja que la que normalmente se reproduce en los manuales escolares. Pero, además de esto, hemos de tener en cuenta que la modelización geométrica del espacio funcional no es más que una parte del modelo, siendo los componentes teóricos que de su aplicación se derivan un excelente compendio explicativo de la organización de las ciudades en un espacio económico competitivo. La teoría de Christáller y las reformulaciones de Berry y Bourne aportaron así un cuerpo teórico y normativo perfectamente actualizado y dotó a los estudios urbanos y territoriales de una serie de conceptos que nos permiten analizar bajo una nueva lógica espacial el funcionamiento del sistema urbano en la organización del territorio. No olvidemos que la red urbana con la modelización del espacio físico, las redes de conectividad y la estructura administrativa siguen siendo el soporte de la ordenación territorial actual, al que se añaden lógicamente otros factores. No es por eso extraño que en los planes de ordenación del territorio de Alemania y de otros países se haga una mención explicita en el capitulo filosófico introductorio de la teoría del Central Place central como modelo teórico en el que debe sustentarse la elaboración del plan nacional, de los planes regionales y de los planes metropolitanos.

Esta comprensión lógica del funcionamiento de las relaciones espaciales ha sido otro valor de la teoría geográfica, y que adquiere una valoración nueva si lo insertamos en el nuevo contexto del sistema urbano global, porque en definitiva esta teoría 
fue la que aportó a la Geografía Urbana y Económica esa lógica espacial, y en esa lógica reside la base para una interpretación del territorio y de la ciudad sumamente útil en la actualidad, tanto para la planificación de las redes urbanas como para la elaboración de escenarios alternativos en las redes de servicios públicos y de distribución en general. Por todas esas razones, frente a valoraciones simplistas, la deficiente interpretación de la teoría que sustenta el modelo exija posiblemente una revisión de los conceptos y del pensamiento geoeocnómico que subyace, y que es lo que le ha proporcionado su mayor interés.

Por orden de la influencia en el desarrollo teórico de la Geografía Urbana pueden situarse las aportaciones de la escuela de ecología urbana de Chicago, de la teoría social de la ocupación del suelo y de los factores que regulan el crecimiento urbano recogido en lo que se llamó Ecología Humana (A.Halley). Al igual que ocurrió en el caso anterior las reproducciones de esa corriente se centraron en lo modelos formales o geométricos, muchas veces sin profundizar en los principios teóricos explicativos que son los que realmente constituyen una importante aportación a la teoría. Probablemente el peso del formalismo paisajístico haya condicionado una sobrevaloración de los esquemas sobre los conceptos, de las formas sobre el pensamiento. Con todo, algunas aplicaciones tempranas pueden señalarse, como el estudio de los procesos de invasión-sucesión y de disimilitud y segregación residencial en el área metropolitana de Bilbao (Precedo 1972) o los diversos estudios que realicé sobre las ciudades gallegas (entre 1990 y 1999) así como la sistematización de la teoría (Estbanez en 1989 con su libro sobre la morfología urbana de las ciudades españolas o en el apartado del manual de geografía humana de 1989). Desde una perspectiva actual esta teoría resulta de indudable interés para la planificación de la ciudad en lo concerniente a la relación entre el crecimiento urbano y la red de transporte público, el mercado de viviendas y el valor de cambio de los espacios residenciales y para el entendimiento de los nuevos procesos que la llegada masiva de inmigrantes de diferentes características está teniendo en la reestructuración social, residencial y comercial de las ciudades españolas; pero tal vez lo más interesante sea su posible utilidad para diseñar escenarios anticipativos para los planes de regulación del crecimiento metropolitano y para el diseño de las redes de transporte público en relación con la demanda previsible.

Una tercera línea de innovación se produjo a partir de la adaptación de las teorías y modelos de economía espacial, tanto urbana como regional. A pesar de las muchas y decisivas transformaciones acontecidas, los supuestos teóricos siguen siendo válidos para temas tales como las políticas de crecimiento y de equilibrio de las áreas y las redes urbanas, la dinámica expansiva de los sistemas de ciudades, y en general para la predicción y anticipación de los procesos de crecimiento urbano acumulativo y de las posibles opciones correctoras o de reequilibrio. Igualmente su interés para el establecimiento de estrategias territoriales de ejes y nodos de desarrollo es grande; 
pero sobre todo ha tenido la virtud de introducir en los estudios urbanos el método científico experimental y facilitar la formulación y comprobación de hipótesis, un tipo de investigación que en los años ochenta la geografía española apenas recogía. En cualquier caso estableció una interesante y fructífera relación entre la geografía urbana y económica y el desarrollo regional en cuyas formulaciones conjuntas, y valoradas con independencia de sus excesos neopositivistas y funcioanlistas, sentaron las bases para lo que después constituiría la planificación estratégica urbana y la teoría de la competencia territorial a partir de las ventajas comparativas o factores diferenciales de desarrollo urbano.

Efectivamente, cuando la crisis industrial de 1973 y la transición postindustrial la aplicación de la planificación estratégica inicial demandaba una conceptualización propia de la ciudad, fue en estas teorías y modelos donde pudo encontrar los apoyos necesarios para su implementación; del mismo modo que el planteamiento de la teoría de la competitividad urbana, de amplia base geográfica, desembocaría en los comienzos del marketing urbano, entendido como aquella actividad cuya finalidad es posicionar las ciudades en el nuevo sistema urbano, definiendo nuevos productos urbanos para diseñar un nuevo modelo de ciudad más competitivo (Precedo 1996). Cuando los planificadores o expertos de citymarketing olvidaron este enfoque, como ha ocurrido en España, el urbanmarketing quedo reducido a una acción tipo postventa subordinada a la planificación estratégica casi siempre excesivamente economicista. Mi experiencia en este tipo de trabajos y la revisión teórica que estoy preparando me ha servido, entre otras cosas, para constatar, una vez más, que la Geografía Urbana tiene una gran oportunidad en este ámbito, y que -como al principio he dicho-, tanto para el análisis intra como interurbano, encuentra aquí un extenso campo de aportaciones para transformar el perfil mercadotécnico actual en una visión más amplia, pensada desde la complejidad social, cultural y económica de la ciudad actual, y al mismo tiempo un banco de experiencias para la planificación participativa y la cocreatividad social (tema que he introduzco recientemente en un articulo sobre este tema).

Otro tema innovador que estimo debe ser resaltado ha sido la teoría de la difusión de las innovaciones de Hägerstrand, no tanto porque generara un incremento de los estudios de este tipo, sino porque situó a la Geografía Urbana en el núcleo conceptual de los que serían los modelos de desarrollo urbano y regional de finales del siglo XX y de principios del s.XXI, particularmente en el estudio de las ciudades intermedias como nodos de conexión de los sistemas regionales con el sistema global para formar el escalón de las metrópolis subglobales de P. Hall dentro de una jerarquía urbana horizontal y desterritorializada, basada en flujos multidireccionales globales, en la línea de lo que estableció Castells.

Para finalizar, me referiré a la cuestión de la información espacial. A partir de los años noventa, y tomando como punto de referencia anterior la aplicación de la fotoin- 
terpretación al estudio de los usos de suelo de los años setenta, irrumpieron entre los geógrafos los sig, cuya novedad y enormes posibilidades orientaron una gran parte de los nuevos trabajos geográficos hacía los sistemas de información y la teledetección, priorizando las dimensiones territoriales de la investigación geográfica con un enfoque ambientalista que fue abriéndose posteriormente hacia in tema transversal como el desarrollo sostenible y el cambio climático. Tal vez sea la preeminencia que en este campo tuvo la geografía física, la planificación medioambiental y la ordenación física del territorio, lo que dejó a la Geografía Urbana actual en un segundo plano, ya que la aplicación de los sig se tradujo casi siempre en un nuevo sistema de clasificación catastral y de los usos del suelo, sin que la necesaria revisión de los conceptos morfológicos y los modelos de crecimiento se haya producido. Quizás también por eso, los estudios actuales de la Geografía Urbana estén subordinados a los temas de interés de los urbanistas de formación arquitectónica, como es el debate entre la urbanización compacta o dispersa, o de los especialistas en la organización administrativa en lo concerniente a la fusión municipal, el gobierno o gobernanza de las áreas metropolitanas y temas de este tipo, en los cuales la especificidad geográfica aunque imprescindible, suele estar supeditada a los enfoques de las disciplinas citadas. Algo parecido a lo que ocurrió con la incursión de los geógrafos en el planeamiento urbanístico y en los centros históricos, en cuyos campos, salvo excepciones (Troitiño con su intervención en Cuenca), nuestra participación ha sido secundaria, aunque no deje de ser una oportunidad para la inserción laboral de los titulados.

A pesar de todo lo dicho, la amplitud y rigurosidad del pensamiento y de la praxis recibida, abre un amplio campo de interés en el que los geógrafos urbanos actuales pueden ir mucho más allá de la práctica para recuperar el campo teórico tal como en las etapas anteriores había ocurrido. Tal vez ese exceso de pragmatismo técnico de la Geografía Urbana actual haya sido lo que le llevó a perder ese carácter innovador que hasta ahora había tendido, y cuya constatación ha sido precisamente uno de los ejes fundamentales de mi argumentación. De todos modos, no debemos olvidar que durante estos últimos años han sido numerosos los estudios que han abordado los problemas sociales de las ciudades, principalmente las desigualdades y la marginalidad, y principalmente la llegada de los nuevos inmigrantes, lo cual ha logrado mantener el espíritu crítico frente al predominio tecnocrático de las técnicas de información geográfica, pero los avances teóricos todavía no han sido sustánciales, subyaciendo amplias reminiscencias de la teoría de Harvey. Otro campo a sistematizar y teorizar bajo una perspectiva global nueva y llena de incertidumbres y problemas, en la cual el neocapitalismo está imponiendo una visión cada vez más alejada de las preocupaciónes propias de la geografía humana.

Me habrán quedado, sin duda, muchos temas y no pocas aportaciones en el tintero, pero lo que he pretendido es poner por escrito una reflexión personal científico-filosófica sobre la evolución y la situación actual de la Geografía Urbana. Solo he 
levantado la vista del ordenador para buscar las fechas que deben acompañar a las citas, y prefiero que este texto mantenga la espontánea viveza de una redacción a vuela pluma, con algo más de diez horas de trabajo detrás, que adoptar el academicismo de un texto normativizado. Por eso tampoco había pensado incluir referencias bibliográficas porque ningún texto he utilizado más allá de lo que mi memoria me iba trayendo al pensamiento, y porque- siendo así- el hacerlo sería como falsear la realidad porque lo único que hice fue poner por escrito mi propio pensamiento y mi experiencia personal. Además, para normativa y burocratización ya nos sobra la actual institución universitaria y esos planes de estudio que apenas dejan tiempo para la reflexión crítica y para la profundización en el pensamiento y en la teoría. Es por ese riesgo por lo que había pensado incidir en la dirección que lo hago: en la contraria al pragmatismo técnico y a la mentalidad indexada dominante entré muchos autores y que la misma arbitrariedad del sistema de medición del conocimiento les impone. Quería así, a través de la heterodoxia, manifestar mi propia y tardía rebeldía ante la situación actual del pensamiento geográfico y en particular de la Geografía Urbana. Pero, una vez más, lo académicamente correcto ha terminado por obligarme a hacerlo.

\section{La geografía urbana hacia el futuro}

Cuando las ciudades tienen que reinventarse para resituarse en el nuevo mapa urbano, y cuando esa reinvención debe abandonar modelos coyunturales basados en el poder de la imagen arquitectónica o de los grandes y costosos eventos por razones del ciclo económico, el análisis geográfico de la ciudad puede constituir un valioso método para identificar los recursos susceptibles de valorización para el diseño del modelo de ciudad o del proyecto-ciudad sobre el que basar su posicionamiento interior y exterior. Indudablemente este modo de ver las cosas desde la óptica de las limitaciones financieras impuestas por la crisis a países como España o a grupos de países como Europa, no tienen por que extenderse a otras latitudes, ya que tanto las nuevas potencias económicas como las ciudades globales de los países emergentes tienen ante si un futuro nada limitante, al contrario, al ser las grandes ciudades los motores de su competitividad global, o dicho de otro modo el soporte físico de la economía global, en ellas la ausencia de limitaciones financieras seguirá primando las grandes inversiones creadoras de imagen, tan deseadas para resituar esas ciudades en el imaginario mundial y también en los mercados globales. Otra cosa es la ética de tal modo de proceder en un mundo donde las desigualdades crecen alarmantemente y donde la pobreza convive al lado de la opulencia más ostentosa y superficial que nunca se había conocido en tiempos modernos. Pero ese no es nuestro caso, y dado que mis reflexiones se refieren a la Geografía Urbana en España las ideas que expuse deben leerse desde ese preciso contexto. 
También las ciudades intermedias tienen que posicionarse y reinventarse para situarse en el nuevo mapa urbano. La base será la diferenciación frente a la homogeneización con una perspectiva comparativa mundial para saber valorar e identificar lo diferente, y en lugar de centrarse solo en lo económico poner el acento en los componentes inmateriales, en la cultura, en la identidad social, en la cohesión de las comunidades territoriales y en otros factores sociales y de calidad de vida. Devolver a la ciudad su naturaleza de un espacio para habitar, para trabajar, para cultivar el cuerpo y el espíritu, como había preconizado Le Courbusier, y no solo para acrecentar el rendimiento económico.

Para esta nueva etapa la Geografía Urbana tiene mucho que aportar pero necesita replantear la valoración de sus contenidos, la perspectiva y decidir y difundir lo que puede aportar para conocer, comprender y mejorar este mundo de ciudades en el que vivimos, superando la amenaza del localismo que, como antes ocurrió, vuelve a dominar la geografía urbana española. Para que los escolares y los especialistas puedan tener un mejor conocimiento del mundo actual ; para construir la ciudad desde la participación social, desde la educación urbana de la sociedad (el empowermnet); para planificar la ciudad con una visón integrada e integradora y para saber discriminar los valores y los recursos urbanos acumulados por la historia urbana y desde ellos diseñar los escenarios futuros, con el fin de ser transmisores cabales de los bienes culturales, materiales e inmateriales, recibidos.

La preocupación de los geógrafos urbanos no debe limitarse, por eso, a una clasificación del suelo como la que hacen los arquitectos, ni a diseñar grandes escaparates, ni a asumir el debate de los márgenes de la ciudad dispersa bajo un enfoque que busca la eficiencia por encima de las aspiraciones culturales y sociales de los individuos y de los grupos sociales; ni una planificación estratégica mecanicista que casi nunca ha dado resultados más allá de la promoción turística o de la imagen, u otras tareas por el estilo. La Geografía Urbana está en condiciones de aportar una perspectiva propia de los procesos que se entrecruzan y se oponen o complementan en el complejo urbano.

La Geografía Urbana está en condiciones de aportar, reuniendo en un compendio teórico revisado, todas las aportaciones anteriores, una profundización en la realidad múltiple dé la ciudad contemporánea, en el conocimiento y explicación de su complejidad y desde una perspectiva más humanista y crítica y menos tecnocrática. Esa será la base para futuras actuaciones y propuestas y para identificar recursos, valores diferenciales y ventajas comparativas, desde una visión internacionalista, tan propia de la primera geografía de las ciudades.

Seguir estudiando casos y situaciones locales o temas sectoriales no hace avanzar la teoría como ya antes se había comprobado. La Geografía Urbana debe recuperar 
su visón global, integradora, sintética y relacional, la visón del geógrafo, para desde ahí contribuir a pensar y a planificar la ciudad en el nuevo contexto, desde un enfoque que ni los economistas, ni los sociólogos, ni los arquitectos, ni los urbanistas administrativistas, pueden aportar. Eso es lo que yo pienso y lo que una variada y prolongada experiencia me ha enseñado; y que por la misma razón he pensado que lo más adecuado sería trasmitirlo en primera persona.

\section{Referencias bibliográficas}

Alomar, G (1980): Teoría de la ciudad. Madrid. IEAL.

Alonso Sanz, J (2004): L'explosió de la citat. Barcelona. COAC.

Arana, J y otros (2003): Ciudades posibles. Madrid. Ed. Lenguadetrapo.

Argan G. C. (1983): Historia del arte como historia de la ciudad. Barcelona. Laia.

López Trigal, L ed.(2003).-La ciudad: nuevos procesos nuevas respuestas .León. A.G.E.

Berry, B (1970): Geographical perspectives on urban system. New Jersey. Englewood Cliffs. Prentice-Hall.

Bielza de Ory, V (2008): Introducción a la ordenación del territorio. Zaragoza. PUZ.

Blanco Fernández, J (2004): La emergencia de las nuevas ciudades en la era global. Oviedo. Trea.

Bourne, L (1990): The changing geography of urban Systems. Pamplona. Eunsa.

Bosque Maurel, J (1962): Geografía Urbana de Granada. Zaragoza. CSIC.

Cabo, A (1981): Personalidad geográfica de una ciudad. Salamanca. EUS.

Capel, H (1974): Estudios sobre el sistema urbano. Ed Universidad de Barcelona.
Capel, H (1975): Capitalismo y morfología urbana en España. Barcelona. Los libros de la Frontera.

Campesino, A. J. (1982): Estructura y paisaje urbano de Cáceres. Cáceres. COAE.

Castell, M (1995): La ciudad informacional. Madrid. Alianza.

Castell, M y Hall, P (1994): Las tecnópolis del mundo .Madrid. Alianza.

Comité Español de la U.G.I (2004): La Geografía española ante los retos de la sociedad actual. Madrid.

Chueca Goitia, F (1968) Breve historia del urbanismo en España. Madrid. Alianza.

Diez Nicolás, J (1972) especialización funcional y dominación en la España urbana. Madrid. Guadarrama.

Elizagarate, V (2003): Marketing de ciudades. Madrid. Pirámide.

Estébanes Álvarez, J (1974): Cuenca: estudio geográfico. Madrid. CSIC.

Estébanez Álvarez (1989): Las ciudades. morfología y estructura. Madrid. Síntesis.

Feria Toribio, J.M. y Albertos J.M. (2010): La ciudad metropolitana en España: procesos urbanos en los inicios del siglo XXI. Pamplona. Civitas. 
Ferrer, M y Precedo A (1977): Las ciudades del Norte de España. Durango. L Zugaza.

Ferrer, M (1992): Los sistemas urbanos. Madrid. Síntesis.

Ferrer, M ed.( 1992):Cambios urbanos y políticas territoriales. Pamplona. Eunsa.

Ferrer, M (ed.) (1995): Perspectivas demográfico-sociales, urbanísticas y territoriales en el umbral del siglo XXI. Pamplona. Eunsa.

Ferrer, M.(2003): Los centros históricos en España. Pamplona. Gobierno de Navarra.

Haggett; P. (1975): Geography: a modern synthesis. New York. Harper Ed.

Garcia Ballesteros, A (1978); Geografía Urbana de Guadalajara. Madrid. F.U.E

Garcia Fernández, J (1974): Crecimiento y estructura urbana de Valladolid Barcelona. Libros de la Frontera.

Gonzalez Dorado,A (1975):Sevilla: centralidad regional y organización interna de su espacio urbano. Barcelona. S.E. Banco Urquijo.

Gozálvez Pérez,V (1976):La ciudad de Elche. Valencia. PUV.

Hawley, A (1966): Ecología Humana. Madrid. Technos.

Hall,P (1996): Ciudades del mañana. Barcelona. Ed del Serbal.

Hall,P ( 2006): Congreso regiones capitales. Madrid. Comunidad de Madrid.

Navarro Ferrer, A. M (1962): Geografía Urbana de Zaragoza. Zaragoza. CSIC.

Precedo, A Ed. (1973) urbanismo. Madrid. BBV.

Panadero Moyá, M. (1976): La ciudad de Albacete. Albacete. Caja de Ahorros.
Precedo Ledo,A. ( 1977). Bilbao y el bajo Nervión: un espacio metropolitano. Bilbao Junta de Cultura de Vizcaya.

Precedo Ledo, A (1988) La red urbana. Madrid. Síntesis.

Precedo Ledo, A (1988): La red urbana de Navarra. Pamplona. C.A.N.

Precedo Ledo, A (1989): Vigo área metropolitana. La Coruña. FCG.

Precedo Ledo, A (1990): La Coruña: metrópoli regional. La Coruña. FCG..

Precedo Ledo, A (1991):.Orense centro de equilibrio. La Coruña. FCG

Precedo Ledo, A (1995): El área urbana de Ferrol: la crisis de un modelo urbano. La Coruña. FCG

Precedo Ledo, A (1996): Ciudad y Desarrollo urbano. Madrid. Síntesis.

Precedo Ledo, A (2004): Nuevas realidades territoriales para el siglo XXI. Madrid. Síntesis.

Precedo A dtor. (2007): El área metropolitana de A Coruña: posicionamiento, constitución y marketing. A Coruña. Diputación Provincial.

Pillet, F. (1984): Geografía Urbana de Ciudad Real. Madrid. Akal.

Puyol, R Ed. (1988) Geografía Humana. Madrid. Síntesis.

Quirós Linares, F.( 1978)El crecimiento espacial de Oviedo. Oviedo Universidad.

Racionero, L. (1978): Sistema de ciudades y ordenación del territorio. Madrid. Alianza.

Romero, J y Farinas J. (eds.) (2006): Gobernanza territorial en España. Valencia. P.U.V.

Serrano Martines, J.M.(2005):Organización y funcionamiento del área metropolitana de Murcia. Murcia. P.U.M. 
Teixidor, MJ. (1982): Valencia: la construció de una ciutat. Valencia. I.A.M..

Torres Balbas, L (1954): Resumen histórico del urbanismo en España. Madrid. IEAL.
Troitiño, M. A (1984): Cuenca: evolución y crisis de una vieja ciudad castellana. Madrid. MOPU.

Zárate, A (1981); Vitoria: transformación y cambio de un espacio urbano. Vitoria. Inst. Sancho el Sabio. 